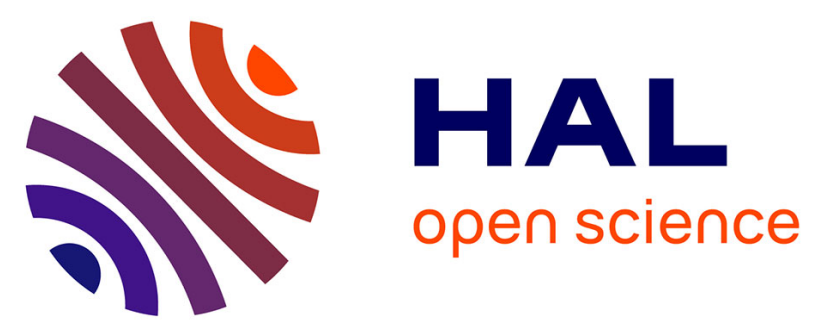

\title{
A Switching Controller for a class of MIMO Bilinear Systems with Time-Delay
}

Tonametl Sanchez, Andrey Polyakov, Emilia Fridman, Laurentiu Hetel

\section{To cite this version:}

Tonametl Sanchez, Andrey Polyakov, Emilia Fridman, Laurentiu Hetel. A Switching Controller for a class of MIMO Bilinear Systems with Time-Delay. IEEE Transactions on Automatic Control, 2020, 65 (5), pp.2250 - 2256. 10.1109/TAC.2019.2940548 . hal-02285126

\section{HAL Id: hal-02285126 \\ https://hal.inria.fr/hal-02285126}

Submitted on 12 Sep 2019

HAL is a multi-disciplinary open access archive for the deposit and dissemination of scientific research documents, whether they are published or not. The documents may come from teaching and research institutions in France or abroad, or from public or private research centers.
L'archive ouverte pluridisciplinaire HAL, est destinée au dépôt et à la diffusion de documents scientifiques de niveau recherche, publiés ou non, émanant des établissements d'enseignement et de recherche français ou étrangers, des laboratoires publics ou privés. 


\title{
A Switching Controller for a class of MIMO Bilinear Systems with Time-Delay
}

\author{
Tonametl Sanchez, Andrey Polyakov, Emilia Fridman, and Laurenţiu Hetel
}

\begin{abstract}
In this paper we propose a state-dependent switching controller for MIMO bilinear systems with constant delays in both the state and the input. The control input is assumed to be restricted to take only a finite number of values. The stability analysis of the closed-loop is based on a Lyapunov-Krasovskii functional, and the design is reduced to solve a system of linear matrix inequalities. The controller can be designed by considering (state) delay-dependent or delay-independent conditions.
\end{abstract}

\section{Index Terms}

Time-delay systems, bilinear systems, switched control.

\section{INTRODUCTION}

In control systems engineering, bilinear systems arise as models of real phenomena from many and diverse areas, e.g. biology, chemistry, physics, social sciences, and engineering [1], [2]. They can also be used to approximate a wide range of more complex nonlinear systems [3], [4]. On the other hand, time-delay is in general an unavoidable phenomenon in control engineering [5], [6], [7]. It can appear as

This work was carried out within the framework of the CNRS Research Federation on Ground Transports and Mobility, in articulation with the ELSAT2020 project supported by the European Community, the French Ministry of Higher Education and Research, the Hauts de France Regional Council. It was also supported by Agence Nationale pour la Recherche DIGITSLID ANR-18-CE40-0008; the Israel Science Foundation, grant No. 1128/14; the Ministry of Education and Science of Russian Federation, project 14.Z50.31.0031; and the Government of Russian Federation, grant 08-08.

T. Sanchez is with Inria, Univ. Lille, CNRS, UMR 9189 - CRIStAL, 59000 Lille, France, and IPICyT, 78216 San Luis Potosí, Mexico, e-mail: tonametl.sanchez@ipicyt.edu.mx

A. Polyakov is with Inria, Univ. Lille, CNRS, UMR 9189 - CRIStAL, 59000 Lille, France, and ITMO University, 197101 St. Petersburg, Russia, e-mail: andrey.polyakov@inria.fr.

E. Fridman is with the Department of Electrical Engineering, Tel-Aviv University, 69978 Tel-Aviv, Israel, e-mail: emilia@eng.tau.ac.il.

L. Hetel is with Centrale Lille, CNRS, UMR 9189 - CRIStAL, 59000 Lille, France, e-mail: laurentiu.hetel@centralelille.fr. 
an inherent feature of the model, or simply due to the lags in sensor or actuator signals. Hence, bilinear delayed systems are worthy of more study to develop general schemes for analysis and control design.

In this paper we design a controller for MIMO bilinear systems with constant delays in both the state and the input. We consider control inputs restricted to take only a finite number of values. This situation arises in several control applications, in particular when on-off actuators are present, e.g. in power electronics [8] and in several pneumatic systems [9]. With this restriction in the control, bilinear systems can also be studied as switched (or piece-wise) affine systems controlled by the switching signal.

It is important to mention that the existing control techniques in related works cannot be applied for the class of systems considered in this paper, e.g.: in [10], [11], [12], [13], [14], bilinear and switched affine systems are studied, but the delayed case is not considered in those papers; the systems studied in [15], [16], [17] are switched and have delay in the input, however, they are linear and the controller is a linear state-feedback; in [18] all the subsystems of the switched system must have a common equilibrium point at the origin and the switching laws are not state-feedback; switched affine delayed systems are studied in [19], [20], [21], however, the systems are assumed to be piecewise linear in a neighbourhood of the origin, and no general switching-control schemes are designed.

In [22], [23], a bilinear differential equation with delays was proposed as a simplified nonlinear model for separated flow control. For a particular case of such a model, a sliding mode controller was proposed in [24]. That controller achieved good performance in different experimental settings. Unfortunately, such a kind of controller was designed only for SISO systems (see also [25]), and its generalization to the MIMO case is not straightforward.

The controller presented in this paper is based on a Lyapunov-Krasovskii functional, and its design reduces to solve a system of linear matrix inequalities (LMIs). A particular case of this controller was announced in [26], but compared with that paper, the following improvements are made in the present one: 1) the design considers now (state) delay-dependent stability conditions, this allows us to enlarge the class of systems that can be controlled; 2) in any case, we consider an arbitrary number of delays in the state for both, the linear and the bilinear terms.

Paper organization: In Section II we describe briefly the control problem. The controller design is explained in Section III. Some simulation examples are shown in Section IV. Concluding remarks and future work are stated in Section V.

Notation: $\mathbb{R}$ and $\mathbb{Z}$ denote the set of real and integer numbers, respectively. For any $a \in \mathbb{R}, \mathbb{R}_{\geq a}$ denotes the set $\{x \in \mathbb{R}: x \geq a\}$. For a matrix $A \in \mathbb{R}^{n \times n}$, we mean by $A>0(A<0)$ that $A$ is positive definite (negative definite). For a finite set $X \subset \mathbb{Z}_{>0}$ and a function $f: X \rightarrow \mathbb{R}, \arg \min _{x \in X}(f(x)):=$ $\{y \in X: f(x) \geq f(y) \forall x \in X\}$. 


\section{Problem Statement}

Consider the following bilinear time-delay system

$$
\dot{x}(t)=A_{0} x(t)+\sum_{k=1}^{N_{1}} A_{k} x\left(t-\tau_{k}\right)+B u(t-\varsigma)+\sum_{s=1}^{N_{2}} \mathcal{A}_{s}(u(t-\varsigma)) x\left(t-\bar{\tau}_{s}\right),
$$

where $\mathcal{A}_{s}(u(t-\varsigma))=\sum_{r=1}^{m} A_{s, r} u_{r}(t-\varsigma), x(t) \in \mathbb{R}^{n}$ is the instantaneous state, $u(t) \in \mathbb{R}^{m}$ is the control input, $A_{0}, A_{k}, A_{s, r} \in \mathbb{R}^{n \times n}, k=1, \ldots, N_{1}, s=1, \ldots, N_{2}, r=1, \ldots, m$, are constant matrices for some finite $N_{1}, N_{2} \in \mathbb{Z}_{\geq 0}$, and $\tau_{k}, \bar{\tau}_{s}, \varsigma \in \mathbb{R}_{\geq 0}$ are constant delays. We consider the output of (1) as the whole instantaneous state $x(t)$ which is accessible for all $t \in \mathbb{R}_{t_{0}}$. We assume the following for (1).

Assumption 1. For any $k$ and any $s$, the delays $\varsigma, \tau_{k}$, and $\bar{\tau}_{s}$ are known and satisfy $0<\varsigma \leq \tau_{k}, \bar{\tau}_{s}$.

The problem we want to solve is stated as follows:

- to design a controller $u$ to track asymptotically a desired constant reference $x^{*} \in \mathbb{R}^{n}$, taking into account that each control component $u_{i}: \mathbb{R} \rightarrow \mathbb{R}$ can only take a finite number of values.

Observe that, under the restriction on the controller, we can only have $N$ different values of the input vector $u$, for some $N \in \mathbb{Z}_{>0}$. If we give a certain order to such $N$ vectors, then we obtain the ordered set $U=\left\{u^{1}, \ldots, u^{N}\right\}$, where each $u^{j} \in \mathbb{R}^{m}, j=1, \ldots, N$, is a constant vector. Also note that, since the control input is bounded, the set of admissible references $x^{*}$ is also bounded in the general case.

Remark 1 (On the existence of solutions). Consider (1) with the initial conditions

$$
x(t)=\phi(t), \quad t \in\left[t_{0}-h, t_{0}\right]
$$

where $h=\max \left(\tau_{1}, \ldots, \tau_{N_{1}}, \bar{\tau}_{1}, \ldots, \bar{\tau}_{N_{2}}\right)$. A function $x: \mathbb{R}_{\geq t_{0}-h} \rightarrow \mathbb{R}^{n}$, that is locally absolutely continuous on $t \in\left[t_{0}, \infty\right)$, is called a solution of problem (1), (2) if it satisfies (1) for almost all $t \in\left[t_{0}, \infty\right)$, and (2) for all $t \leq t_{0}$, see e.g. [27], [28], [29, Definition B.1]. If $u:\left[t_{0}-\varsigma, \infty\right) \rightarrow \mathbb{R}^{m}$ is a Lebesgue-measurable and locally essentially bounded function, then (1) satisfies the Carathéodory conditions [27, p. 58], [28, p. 100]. Hence, for any continuous function ${ }^{1} \phi:\left[t_{0}-h, t_{0}\right) \rightarrow \mathbb{R}^{n}$, there exists a unique solution of the problem (1), (2), see e.g. [27, p. 58], [28, p. 100]. Recall that the solution of the problem (1), (2) can be obtained by means of the method of steps (see e.g. [28, p. 89]), i.e. by solving the problem (1), (2) for $t$ in the intervals $\left[t_{0}, t_{0}+\varsigma\right],\left[t_{0}+\varsigma, t_{0}+2 \varsigma\right]$, and so on. Since $u$ is considered as a Lebesgue-measurable function, the integrals in this paper are understood in the sense of Lebesgue.

${ }^{1}$ For (1), the conditions (on the initial function) that guarantee existence and uniqueness of solutions can be relaxed, i.e. $\phi$ can be assumed to be a Borel-measurable bounded function [29, Theorem B.1]. 


\section{A. Reformulation of the problem and the admissible references}

To solve the problem established in the previous section, we first state some basic preliminary considerations.

For $j=1, \ldots, N$ and $s=1, \ldots, N_{2}$, define the vectors $B_{j}=B u^{j}$ and the matrices $\mathcal{A}_{s, j}=$ $\sum_{r=1}^{m} A_{s, r} u_{r}^{j}$. Hence, we can consider (1) as an affine switched system given by

$$
\dot{x}(t)=A_{0} x(t)+\sum_{k=1}^{N_{1}} A_{k} x\left(t-\tau_{k}\right)+\sum_{s=1}^{N_{2}} \mathcal{A}_{s, \sigma(t-\varsigma)} x\left(t-\bar{\tau}_{s}\right)+B_{\sigma(t-\varsigma)},
$$

where $\sigma: \mathbb{R} \rightarrow\{1, \ldots, N\}$ is the switching signal. As stated in Section II, the control objective is to track asymptotically a constant reference $x^{*} \in \mathbb{R}^{n}$. Define the tracking error $z$ by means of the change of coordinates $z(t)=x(t)-x^{*}$. Hence, the tracking error dynamics is given by

$$
\dot{z}(t)=A_{0} z(t)+\sum_{k=1}^{N_{1}} A_{k} z\left(t-\tau_{k}\right)+\sum_{s=1}^{N_{2}} \mathcal{A}_{s, \sigma(t-\varsigma)} z\left(t-\bar{\tau}_{s}\right)+C(t),
$$

where $C(t):=\left(A_{0}+\sum_{k=1}^{N_{1}} A_{k}+\sum_{s=1}^{N_{2}} \mathcal{A}_{s, \sigma(t-\varsigma)}\right) x^{*}+B_{\sigma(t-\varsigma)}$. Thus, we have changed the original problem of designing the controller $u$ to the problem of designing the switching rule for $\sigma$ to drive the trajectories of (3) to the reference $x^{*}$, or equivalently, to drive the trajectories of (4) to zero.

The following lemma gives a necessary condition for the selection of a reference point $x^{*}$.

Lemma 1. Consider (4) and define the following

$$
\begin{aligned}
& \Gamma=\left\{\gamma \in \mathbb{R}^{N}: 0 \leq \gamma_{i} \leq 1, \sum_{j=1}^{N} \gamma_{j}=1\right\} \\
& \overline{\mathcal{A}}_{s}(\gamma)=\sum_{j=1}^{N} \gamma_{j} \mathcal{A}_{s, j}, \quad \bar{B}(\gamma)=\sum_{j=1}^{N} \gamma_{j} B_{j} .
\end{aligned}
$$

If for a given signal $\sigma: \mathbb{R} \rightarrow\{1, \ldots, N\}$ and a constant $x^{*} \in \mathbb{R}, \bar{z}$ is a solution of (4) such that $\bar{z}(t) \rightarrow 0$ as $t \rightarrow \infty$, then there exists $\gamma \in \Gamma$ such that

$$
\left(A_{0}+\sum_{k=1}^{N_{1}} A_{k}+\sum_{s=1}^{N_{2}} \overline{\mathcal{A}}_{s}(\gamma)\right) x^{*}+\bar{B}(\gamma)=0
$$

Proof. For $t>t_{0}$ and any $T \in \mathbb{R}_{>0}$ we have from (4) that

$$
\begin{array}{r}
\bar{z}(t+T)-\bar{z}(t)=\int_{t}^{t+T}\left(A_{0} \bar{z}(\nu)+\sum_{k=1}^{N_{1}} A_{k} \bar{z}\left(\nu-\tau_{k}\right)+\right. \\
\left.\sum_{s=1}^{N_{2}} \mathcal{A}_{s, \sigma(t-\varsigma)} \bar{z}\left(\nu-\bar{\tau}_{s}\right)\right) \mathrm{d} \nu+\int_{t}^{t+T} C(\nu) \mathrm{d} \nu
\end{array}
$$

According to the hypothesis of the lemma, for any finite $T \in \mathbb{R}_{>0}, \lim _{t \rightarrow \infty}(\bar{z}(t+T)-\bar{z}(t))=0$, and

$$
\lim _{t \rightarrow \infty} \int_{t}^{t+T}\left(A_{0} \bar{z}(\nu)+\sum_{k=1}^{N_{1}} A_{k} \bar{z}\left(\nu-\tau_{k}\right)+\sum_{s=1}^{N_{2}} \mathcal{A}_{s, \sigma(t-\varsigma)} \bar{z}\left(\nu-\bar{\tau}_{s}\right)\right) \mathrm{d} \nu=0,
$$


therefore, it is necessary that $\lim _{t \rightarrow \infty} \int_{t}^{t+T} C(\nu) \mathrm{d} \nu=0$ for any finite $T \in \mathbb{R}_{>0}$. Note that, according to the definition of $\mathcal{A}_{s, \sigma(t-\varsigma)}$ and $B_{\sigma(t-\varsigma)}$, we have that

$$
\begin{aligned}
\lim _{t \rightarrow \infty} \int_{t}^{t+T} C(\nu) \mathrm{d} \nu= & \lim _{t \rightarrow \infty}\left(\sum _ { j = 1 } ^ { N } \left[\left(A_{0}+\sum_{k=1}^{N_{1}} A_{k}+\right.\right.\right. \\
& \left.\left.\left.\sum_{s=1}^{N_{2}} \sum_{r=1}^{m} A_{s, r} u_{r}^{j}\right) x^{*}+B u^{j}\right] \delta_{j}(t, T)\right),
\end{aligned}
$$

where $\delta_{j}(t, T)$ is the measure of the set $\left\{\tau \in[t, t+T]: u(\tau-\varsigma)=u^{j}\right\}$. Observe that for any $t \in \mathbb{R}_{>t_{0}}$, $\sum_{j=1}^{N} \delta_{j}(t, T)=T$, thus,

$$
0=\lim _{t \rightarrow \infty} \int_{t}^{t+T} C(\nu) \mathrm{d} \nu=T \sum_{j=1}^{N} \gamma_{j}\left[\left(A_{0}+\sum_{k=1}^{N_{1}} A_{k}+\sum_{s=1}^{N_{2}} \sum_{r=1}^{m} A_{s, r} u_{r}^{j}\right) x^{*}+B u^{j}\right],
$$

where $\gamma_{j}=T^{-1} \lim _{t \rightarrow \infty} \delta_{j}(t, T)$. Observe that $\sum_{j=1}^{N} \gamma_{j}=1$. The result of the lemma is obtained by noticing that $\sum_{j=1}^{N} \gamma_{j}\left[\left(A_{0}+\sum_{k=1}^{N_{1}} A_{k}+\sum_{s=1}^{N_{2}} \sum_{r=1}^{m} A_{s, r} u_{r}^{j}\right) x^{*}+B u^{j}\right]=\left(A_{0}+\sum_{k=1}^{N_{1}} A_{k}+\right.$ $\left.\sum_{s=1}^{N_{2}} \overline{\mathcal{A}}_{s}(\gamma)\right) x^{*}+\bar{B}(\gamma)$.

\section{Controller DESign}

In this section we design two switching laws for $\sigma$ to solve the problem established in the previous section. With the aim of showing the ideas in a clear way, we begin by stating the results for the particular case of (3) with $N_{1}=0$ and $N_{2}=1$, i.e. for the system

$$
\dot{x}(t)=A_{0} x(t)+A_{\sigma(t-\varsigma)} x(t-\tau)+B_{\sigma(t-\varsigma)} .
$$

The results given in Section III-A are extended, in Section III-B, for the case of arbitrary $N_{1}, N_{2}$.

\section{A. One delay in the state}

In the following theorem, we give a switching law $\sigma$ that drives the trajectories of (6) to a given reference $x^{*}$. Such a switching law is based on a Lyapunov-Krasovskii functional that provides delaydependent stability conditions (regarding the delay in the state). As a particular case, delay-independent stability conditions are obtained.

Theorem 1 (Delay-dependent conditions). Consider (6) with $\tau>\varsigma>0$, and a given $x^{*} \in \mathbb{R}^{n}$. If there exist matrices $P_{1}, P_{2}, P_{3}, S, R \in \mathbb{R}^{n \times n}$ and a vector $\gamma \in \Gamma$, such that $P_{1}, P_{3}, S, R$ are symmetric, $\left(A_{0}+\bar{A}(\gamma)\right) x^{*}+\bar{B}(\gamma)=0$,

$$
S, R>0, \quad\left[\begin{array}{cc}
P_{1} & P_{2} \\
P_{2}^{\top} & P_{3}+\tau^{-1} S
\end{array}\right]>0, \quad \bar{M}(\gamma)<0,
$$


where $\bar{A}(\gamma)=\sum_{j=1}^{N} \gamma_{j} A_{j}$,

$$
\bar{M}(\gamma):=\left[\begin{array}{ccc}
\Phi & P_{1} \bar{A}(\gamma)-P_{2} & \tau\left(A_{0}^{\top} P_{2}+P_{3}\right) \\
* & -S & \tau\left(\bar{A}(\gamma)^{\top} P_{2}-P_{3}\right) \\
* & * & -\tau R
\end{array}\right],
$$

and $\Phi:=A_{0}^{\top} P_{1}+P_{1}^{\top} A_{0}+S+\tau R+P_{2}+P_{2}^{\top}$. Then, (6) in closed-loop with the switching controller

$$
\begin{aligned}
\sigma(t) & \in \underset{j \in\{1, \ldots, N\}}{\arg \min }\left(\left[\left(w(t)-x^{*}\right)^{\top} P_{1}+\left(\bar{w}(t)-x^{*} \tau\right)^{\top} P_{2}^{\top}\right]\left[A_{j} x(t-\tau+\varsigma)+B_{j}\right]\right), \\
w(t) & =\int_{-\varsigma}^{0} \mathrm{e}^{-A_{0} \eta}\left[A_{\sigma(t+\eta)} x(t-\tau+\varsigma+\eta)+B_{\sigma(t+\eta)}\right] \mathrm{d} \eta+\mathrm{e}^{A_{0} \varsigma} x(t), \\
\bar{w}(t) & =\int_{t-\tau+\varsigma}^{t} x(\nu) \mathrm{d} \nu+\int_{t-\varsigma}^{t} w(\nu) \mathrm{d} \nu,
\end{aligned}
$$

is such that $x(t) \rightarrow x^{*}$ as $t \rightarrow \infty$.

The proof of Theorem 1 is given below, but first, let us enunciate some important remarks.

Remark 2. Observe that, for any $t \in \mathbb{R}_{\geq 0}$, the switching signal $\sigma(t)$ depends on $x(\vartheta), \vartheta \in[t-\tau, t]$, and the switching signal $\sigma(t-\varsigma)$ depends on $x(\vartheta), \vartheta \in[t-\tau-\varsigma, t-\varsigma]$. Therefore, the condition $\varsigma>0$ allows us to guarantee the existence of solutions of the closed-loop (6), (10) as stated in Remark 1. Thus, no generalized-solution concepts are required.

From Theorem 1 we recover in the following corollary the controller designed in [26].

Corollary 1 (Delay-independent conditions). Consider (6) with $\tau>\varsigma>0$, and a given $x^{*} \in \mathbb{R}^{n}$. If there exist $\gamma \in \Gamma$ and symmetric matrices $P_{1}, S \in \mathbb{R}^{n \times n}$ such that $\left(A_{0}+\bar{A}(\gamma)\right) x^{*}+\bar{B}(\gamma)=0$,

$$
P_{1}, S>0, \bar{M}(\gamma):=\left[\begin{array}{cc}
P_{1} A_{0}+A_{0}^{\top} P_{1}+S & P_{1} \bar{A}(\gamma) \\
\bar{A}^{\top}(\gamma) P_{1} & -S
\end{array}\right]<0,
$$

then (6) in closed-loop with the switching controller

$$
\begin{aligned}
& \sigma(t) \in \underset{j \in\{1, \ldots, N\}}{\arg \min }\left(\left(w(t)-x^{*}\right) P_{1}\left[A_{j} x(t-\tau+\varsigma)+B_{j}\right]\right), \\
& w(t)=\int_{-\varsigma}^{0} \mathrm{e}^{-A_{0} \eta}\left[A_{\sigma(t+\eta)} x(t-\tau+\varsigma+\eta)+B_{\sigma(t+\eta)}\right] \mathrm{d} \eta+\mathrm{e}^{A_{0} \varsigma} x(t),
\end{aligned}
$$

is such that $x(t) \rightarrow x^{*}$ as $t \rightarrow \infty$.

Remark 3. Observe that to accomplish the last LMI in (9), $A_{0}$ must be Hurwitz. Such a restriction is eliminated by the more general controller (8), which considers delay-dependent stability conditions. However, for the cases where both controllers are feasible, (10) has the advantage to be simpler than (8). 
Remark 4 (Two computational issues). Let us discuss briefly two details in the computation of the controller given in Theorem 1:

1) In applications, the controller implementation is normally made through digital computers, thus, the control signals are assumed constant between the sampling periods (piecewise continuous), and the data acquisition process usually transforms the physical measurements into piecewise continuous signals. For such a kind of signals, the Lebesgue and the Riemann integrals coincide. Under this consideration, the integral in the controller of Theorem 1 can be implemented as a Riemann integral.

2) For the computation of the predictors in the control law, there exist in the literature several numerically stable methods to implement such predictors, see e.g. [30], [31], and the references therein.

Proof of Theorem 1: Consider again the change of variables $z(t)=x(t)-x^{*}$. The dynamics in variable $z$ is given by

$$
\dot{z}(t)=A_{0} z(t)+A_{\sigma(t-\varsigma)} z(t-\tau)+C(t),
$$

where $C(t):=\left(A_{0}+A_{\sigma(t-\varsigma)}\right) x^{*}+B_{\sigma(t-\varsigma)}$. The proof consists in verifying, by means of a LyapunovKrasovskii functional, that the solutions of (11) converge asymptotically to zero.

Consider the following Lyapunov-Krasovskii functional [32]

$$
\begin{aligned}
V\left(z_{t}\right)= & {\left[\begin{array}{l}
z(t) \\
\hat{z}(t)
\end{array}\right]^{\top}\left[\begin{array}{cc}
P_{1} & P_{2} \\
* & P_{3}
\end{array}\right]\left[\begin{array}{l}
z(t) \\
\hat{z}(t)
\end{array}\right]+\int_{t-\tau}^{t} z^{\top}(\nu) \times } \\
& R z(\nu)(\nu-t+\tau) \mathrm{d} \nu+\int_{t-\tau}^{t} z^{\top}(\nu) S z(\nu) \mathrm{d} \nu,
\end{aligned}
$$

where $\hat{z}(t):=\int_{t-\tau}^{t} z(\nu) \mathrm{d} \nu$. By using the Jensen's inequality in the last term of (12) we have that

$$
V\left(z_{t}\right) \geq\left[\begin{array}{c}
z(t) \\
\hat{z}(t)
\end{array}\right]^{\top}\left[\begin{array}{cc}
P_{1} & P_{2} \\
P_{2}^{\top} & P_{3}+\tau^{-1} S
\end{array}\right]\left[\begin{array}{c}
z(t) \\
\hat{z}(t)
\end{array}\right]+\int_{t-\tau}^{t}(\nu-t+\tau) z^{\top}(\nu) R z(\nu) \mathrm{d} \nu .
$$

Thus, (12) is positive definite if

$$
R>0, \quad \text { and } \quad\left[\begin{array}{cc}
P_{1} & P_{2} \\
P_{2}^{\top} & P_{3}+\tau^{-1} S
\end{array}\right]>0 .
$$

Since the solutions of the closed-loop (6), (8) are absolutely continuous functions (see Remarks 1 and 
2), the time derivative of (12) is given by (for almost all $\left.t \in \mathbb{R}_{\geq t_{0}}\right)^{2}$

$$
\begin{aligned}
\dot{V}\left(z_{t}\right)= & 2\left[\begin{array}{c}
z(t) \\
\hat{z}(t)
\end{array}\right]^{\top}\left[\begin{array}{cc}
P_{1} & P_{2} \\
* & P_{3}
\end{array}\right]\left[\begin{array}{c}
\dot{z}(t) \\
\dot{z}(t)
\end{array}\right]+ \\
& \tau z^{\top}(t) R z(t)+z^{\top}(t) S z(t)-z^{\top}(t-\tau) \times \\
& S z(t-\tau)-\int_{t-\tau^{t}}^{t} z^{\top}(\nu) R z(\nu) \mathrm{d} \nu .
\end{aligned}
$$

By using the Jensen's inequality in the last term of (13) we have that $\dot{V}\left(z_{t}\right) \leq W(\zeta ; \sigma(t-\varsigma))$, where $\zeta=\left[z^{\top}(t), z^{\top}(t-\tau), \hat{z}^{\top}(t)\right]^{\top}$ and

$$
\begin{aligned}
W(\zeta ; \sigma(t-\varsigma))= & z^{\top}(t) \Phi z(t)+2 z^{\top}(t)\left(P_{1} A_{\sigma(t-\varsigma)}-P_{2}\right) z(t-\tau)+2 z^{\top}(t)\left(A_{0}^{\top} P_{2}+P_{3}\right) \hat{z}(t)- \\
& z^{\top}(t-\tau) S z(t-\tau)+2 z^{\top}(t-\tau)\left(A_{\sigma(t-\varsigma)}^{\top} P_{2}-P_{3}\right) \hat{z}(t)-\tau^{-1} \hat{z}^{\top}(t) R \hat{z}+ \\
& 2\left(z^{\top}(t) P_{1}+\hat{z}^{\top}(t) P_{2}^{\top}\right) C(t) .
\end{aligned}
$$

Observe that (14) can be rewritten as $W(\zeta ; \sigma(t-\varsigma))=\bar{\zeta}^{\top} M(\sigma(t-\varsigma)) \bar{\zeta}+2\left(z^{\top}(t) P_{1}+\hat{z}^{\top}(t) P_{2}^{\top}\right) C(t)$, where $\bar{\zeta}=\left[z^{\top}(t), z^{\top}(t-\tau), \tau^{-1} \hat{z}^{\top}(t)\right]^{\top}$ and

$$
M(\rho)=\left[\begin{array}{ccc}
\Phi & P_{1} A_{\rho}-P_{2} & \tau\left(A_{0}^{\top} P_{2}+P_{3}\right) \\
* & -S & \tau\left(A_{\rho}^{\top} P_{2}-P_{3}\right) \\
* & * & -\tau R
\end{array}\right] .
$$

Now, define the function $W_{\gamma}: \mathbb{R}^{3 n} \rightarrow \mathbb{R}$, given by $W_{\gamma}(\zeta)=\bar{\zeta}^{\top} \bar{M}(\gamma) \bar{\zeta}+2\left(z^{\top}(t) P_{1}+\hat{z}^{\top}(t) P_{2}^{\top}\right)\left[\left(A_{0}+\right.\right.$ $\left.\bar{A}(\gamma)) x^{*}+\bar{B}(\gamma)\right]$. Note that, according to the hypotheses of the theorem, $W_{\gamma}$ is negative definite, and can be rewritten as

$$
W_{\gamma}(\zeta)=\sum_{j=1}^{N} \gamma_{j} W(\zeta ; j)
$$

Since $W_{\gamma}$ is negative definite, and each $\gamma_{i}$ is nonnegative, we can assure that for each $\zeta \in \mathbb{R}^{3 n} \backslash\{0\}$, there exists (at least) one $j \in\{1, \ldots, N\}$ such that $W(\zeta ; j)<0$. Therefore, the switching law $\sigma(t-\varsigma) \in$

\footnotetext{
${ }^{2}$ In general, the right-hand side of the closed-loop (6), (8) is not continuous in $t$, however, its solutions are absolutely continuous in $t$, as stated in Remarks 1 and 2. Thus, the smoothness properties of the functional $V$ guarantee that its evaluation along a system's trajectory is an absolutely continuous function, therefore, its time derivative exists almost everywhere. Hence, the standard Lyapunov-Krasovskii theorems are still valid for our case. Nevertheless, there exist some stability results where Carathéodory conditions and non-differentiable Lyapunov-Krasovskii functionals are considered, see e.g. [33].
} 
$\arg \min _{j \in\{1, \ldots, N\}} W(\zeta ; j)$ ensures that (14) is strictly negative for all $\zeta \in \mathbb{R}^{3 n} \backslash\{0\}$. However, observe that (see (14))

$$
\begin{array}{r}
\min _{j} W(\zeta ; j)=2 z^{\top}(t) \Phi z(t)-2 z^{\top}(t-\tau) P_{2} z(t)+2 z^{\top}(t) \times \\
\left(A_{0}^{\top} P_{2}+P_{3}\right) \hat{z}(t)-z^{\top}(t-\tau) S z(t-\tau)-2 z^{\top}(t-\tau) \times \\
P_{3} \hat{z}(t)-\tau^{-1} \hat{z}^{\top}(t) R \hat{z}(t)+2\left(z^{\top}(t) P_{1}+\hat{z}^{\top}(t) P_{2}^{\top}\right) \times \\
A_{0} x^{*}+2 \min _{j \in\{1, \ldots, N\}}\left\{\left(z^{\top}(t) P_{1}+\hat{z}^{\top}(t) P_{2}^{\top}\right) \times\right. \\
\left.\left(A_{j} z(t-\tau)+A_{j} x^{*}+B_{j}\right)\right\} .
\end{array}
$$

Hence, the switching rule $\sigma(t-\varsigma) \in \arg \min _{j \in\{1, \ldots, N\}} W(\zeta ; j)$ is equivalent to

$$
\sigma(t) \in \underset{j \in\{1, \ldots, N\}}{\arg \min }\left\{\left(z^{\top}(t+\varsigma) P_{1}+\hat{z}^{\top}(t+\varsigma) P_{2}^{\top}\right)\left(A_{j} z(t-\tau+\varsigma)+A_{j} x^{*}+B_{j}\right)\right\} .
$$

But $\sigma$ requires the prediction of the instantaneous state at $t+\varsigma$. Since the solution of (6) can be written as

$$
x(t)=\int_{t_{0}}^{t} \mathrm{e}^{A_{0}(t-\nu)}\left[A_{\sigma(\nu-\varsigma)} x(\nu-\tau)+B_{\sigma(\nu-\varsigma)}\right] \mathrm{d} \nu+\mathrm{e}^{A_{0}\left(t-t_{0}\right)} x\left(t_{0}\right),
$$

the prediction of the state at $t+\varsigma$ is given by

$$
x(t+\varsigma)=\int_{t}^{t+\varsigma} \mathrm{e}^{A_{0}(t+\varsigma-\nu)}\left[A_{\sigma(\nu-\varsigma)} x(\nu-\tau)+B_{\sigma(\nu-\varsigma)}\right] \mathrm{d} \nu+\mathrm{e}^{A_{0} \varsigma} x(t) .
$$

Observe that $w(t)=x(t+\varsigma)$ by means of the change of variable $\eta=-t-\varsigma+\nu$. Moreover, we have that $\hat{z}^{\top}(t+\varsigma)=\int_{t-\tau+\varsigma}^{t} x(\nu) \mathrm{d} \nu+\int_{t}^{t+\varsigma} x(\nu) \mathrm{d} \nu-x^{*} \tau$. Thus, the controller is obtained by using back the change of coordinates.

Remark 5. Note that at the time $t$, the predictor used to compute $x(t+\varsigma)$ only requires past information, i.e. the values of $x(r)$ for $r \in[t-\tau, t)$ (which are already measured), and the values of $\sigma(r)$ for $r \in[t-\varsigma, t)$ (which are already computed).

Finally, note that the switching law ensures that

$$
W(\zeta ; \sigma(t-\varsigma))=\min _{j \in\{1, \ldots, N\}} W(\zeta ; j)=\sum_{j^{\prime}=1}^{N} \gamma_{j^{\prime}} \min _{j \in\{1, \ldots, N\}} W(\zeta ; j) \leq \sum_{j=1}^{N} \gamma_{j} W(\zeta ; j)=W_{\gamma}(\zeta) .
$$

Thus, $\dot{V}\left(x_{t}\right) \leq W_{\gamma}(\zeta)=\bar{\zeta}^{\top} \bar{M}(\gamma) \bar{\zeta} \leq-\lambda_{\min }(-\bar{M}(\gamma))|z(t)|^{2}$, where $|\cdot|$ denotes the Euclidean norm. To prove Corollary 1 , just set $R=P_{2}=P_{3}=0$ in the functional (12), and follow the same reasoning. 


\section{B. Several delays in the state}

In this section we extend the results given in Section III-A to the class of systems described by (3) considering several delays in the state. For this case consider the matrices $P_{1}, P_{2, k}, \bar{P}_{2, s}, P_{3, k}, \bar{P}_{3, s}, S_{k}, \bar{S}_{s}, R_{k}, \bar{R}_{s} \in$ $\mathbb{R}^{n \times n}, k=1, \ldots, N_{1}, s=1, \ldots, N_{2}$. Define

$$
\bar{P}=\left[\begin{array}{cc}
P_{1} & \Pi_{11} \\
* & \Pi_{22}
\end{array}\right], \quad \bar{M}(\gamma)=\left[\begin{array}{ccc}
\Phi_{11} & \cdots & \Phi_{15} \\
& \ddots & \vdots \\
* & & \Phi_{55}
\end{array}\right],
$$

where

$$
\begin{aligned}
& \Pi_{11}=\left[\begin{array}{llllll}
P_{2,1} & \cdots & P_{2, N_{1}} & \bar{P}_{2,1} & \cdots & \bar{P}_{2, N_{2}}
\end{array}\right], \\
& \Pi_{22}=\operatorname{diag}\left(P_{3,1}+\tau_{1}^{-1} S_{1}, \ldots, P_{3, N_{1}}+\tau_{N_{1}}^{-1} S_{N_{1}}, \bar{P}_{3,1}+\bar{\tau}_{1}^{-1} \bar{S}_{1}, \ldots, \bar{P}_{3, N_{2}}+\bar{\tau}_{N_{2}}^{-1} \bar{S}_{N_{2}}\right), \\
& \Phi_{11}=A_{0}^{\top} P_{1}+P_{1}^{\top} A_{0}+\sum_{k=1}^{N_{1}}\left(\tau_{k} R_{k}+S_{k}+P_{2, k}+P_{2, k}^{\top}\right)+\sum_{s=1}^{N_{2}}\left(\bar{\tau}_{s} \bar{R}_{s}+\bar{S}_{s}+\bar{P}_{2, s}+\bar{P}_{2, s}^{\top}\right), \\
& \Phi_{12}=\left[P_{1} A_{1}-P_{2,1} \cdots P_{1} A_{N_{1}}-P_{2, N_{1}}\right], \\
& \Phi_{13}=\left[P_{1} \overline{\mathcal{A}}_{1}(\gamma)-\bar{P}_{2,1} \cdots P_{1} \overline{\mathcal{A}}_{N_{2}}(\gamma)-\bar{P}_{2, N_{2}}\right] \text {, } \\
& \Phi_{14}=\left[A_{0}^{\top} P_{2,1}+P_{3,1} \cdots A_{0}^{\top} P_{2, N_{1}}+P_{3, N_{1}}\right], \\
& \Phi_{15}=\left[A_{0}^{\top} \bar{P}_{2,1}+\bar{P}_{3,1} \cdots A_{0}^{\top} \bar{P}_{2, N_{2}}+\bar{P}_{2, N_{2}}\right], \\
& \Phi_{22}=-\operatorname{diag}\left(S_{1}, \ldots, S_{N_{1}}\right), \quad \Phi_{23}=0, \\
& \Phi_{24}=\left[\begin{array}{cccc}
\varphi_{1} & A_{1}^{\top} P_{2,2} & \cdots & A_{1}^{\top} P_{2, N_{1}} \\
A_{2}^{\top} P_{2,1} & \varphi_{2} & \cdots & A_{2}^{\top} P_{2, N_{1}} \\
\vdots & & \ddots & \vdots \\
A_{N_{1}}^{\top} P_{2,1} & & \cdots & \varphi_{N_{1}}
\end{array}\right],
\end{aligned}
$$

with $\varphi_{k}=A_{k}^{\top} P_{2, k}-P_{3, k}$,

$$
\begin{aligned}
\Phi_{25} & =\left[\begin{array}{ccc}
A_{1}^{\top} \bar{P}_{2,1} & \cdots & A_{1}^{\top} \bar{P}_{2, N_{2}} \\
\vdots & \ddots & \vdots \\
A_{N_{1}}^{\top} \bar{P}_{2,1} & \cdots & A_{N_{1}}^{\top} \bar{P}_{2, N_{2}}
\end{array}\right], \\
\Phi_{33} & =-\operatorname{diag}\left(\bar{S}_{1}, \ldots, \bar{S}_{N_{2}}\right), \\
\Phi_{34} & =\left[\begin{array}{ccc}
\overline{\mathcal{A}}_{1}^{\top}(\gamma) P_{2,1} & \cdots & \overline{\mathcal{A}}_{1}^{\top}(\gamma) P_{2, N_{1}} \\
\vdots & \ddots & \vdots \\
\overline{\mathcal{A}}_{N_{2}}^{\top}(\gamma) P_{2,1} & \cdots & \overline{\mathcal{A}}_{N_{2}}^{\top}(\gamma) P_{2, N_{1}}
\end{array}\right],
\end{aligned}
$$




$$
\Phi_{35}=\left[\begin{array}{cccc}
\bar{\varphi}_{1} & \overline{\mathcal{A}}_{1}^{\top}(\gamma) \bar{P}_{2,2} & \cdots & \overline{\mathcal{A}}_{1}^{\top}(\gamma) \bar{P}_{2, N_{2}} \\
\overline{\mathcal{A}}_{2}^{\top}(\gamma) \bar{P}_{2,1} & \bar{\varphi}_{2} & \cdots & \overline{\mathcal{A}}_{2}^{\top}(\gamma) \bar{P}_{2, N_{2}} \\
\vdots & & \ddots & \vdots \\
\overline{\mathcal{A}}_{N_{2}}^{\top}(\gamma) \bar{P}_{2,1} & & \cdots & \bar{\varphi}_{N_{2}}
\end{array}\right]
$$

with $\bar{\varphi}_{s}=\overline{\mathcal{A}}_{s}^{\top}(\gamma) \bar{P}_{2, s}-\bar{P}_{3, s}$,

$$
\begin{aligned}
& \Phi_{44}=-\operatorname{diag}\left(\tau_{1}^{-1} R_{1}, \ldots, \tau_{N_{1}}^{-1} R_{N_{1}}\right), \quad \Phi_{45}=0, \\
& \Phi_{55}=-\operatorname{diag}\left(\bar{\tau}_{1}^{-1} \bar{R}_{1}, \ldots, \bar{\tau}_{N_{2}}^{-1} \bar{R}_{N_{2}}\right) .
\end{aligned}
$$

Theorem 2. Consider (3) satisfying Assumption 1, and a given $x^{*} \in \mathbb{R}^{n}$. If there exist $\gamma \in \Gamma$, and matrices $P_{1}, P_{2, k}, \bar{P}_{2, s}, P_{3, k}, \bar{P}_{3, s}, S_{k}, \bar{S}_{s}, R_{k}, \bar{R}_{s}$, such that (5) holds, $P_{1}, P_{3, k}, \bar{P}_{3, s}, S_{k}, \bar{S}_{s}, R_{k}, \bar{R}_{s}$ are symmetric, $S_{k}, \bar{S}_{s}, R_{k}, \bar{R}_{s}, \bar{P}>0$, and $\bar{M}(\gamma)<0$, then (3) in closed-loop with the switching controller

$$
\sigma(t) \in \underset{j \in\{1, \ldots, N\}}{\arg \min }\left\{\left(\Delta_{1}+\Delta_{2}+\Delta_{3}\right)\left(\Delta_{4}(j)+B_{j}\right)\right\},
$$

with $\Delta_{1}:=\left(w(t)-x^{*}\right)^{\top} P_{1}, \Delta_{2}:=\sum_{k=1}^{N_{1}}\left(\hat{w}_{k}(t)-x^{*} \tau_{k}\right)^{\top} P_{2, k}^{\top}, \Delta_{3}:=\sum_{s=1}^{N_{2}}\left(\hat{\bar{w}}_{s}(t)-x^{*} \bar{\tau}_{s}\right)^{\top} \bar{P}_{2, s}^{\top}$, $\Delta_{4}(j):=\sum_{\tilde{s}=1}^{N_{2}} \mathcal{A}_{\tilde{s}, j} x\left(t-\bar{\tau}_{\tilde{s}}+\varsigma\right)$

$$
\begin{array}{r}
w(t):=\mathrm{e}^{A_{0} \varsigma} x(t)+\int_{-\varsigma}^{0} \mathrm{e}^{-A_{0} \eta}\left(\sum_{k=1}^{N_{1}} A_{k} x\left(t-\tau_{k}+\varsigma+\eta\right)+\right. \\
\left.\sum_{s=1}^{N_{2}} \mathcal{A}_{s, \sigma(t+\eta)} x\left(t-\bar{\tau}_{s}+\varsigma+\eta\right)+B_{\sigma(t+\eta)}\right) \mathrm{d} \eta,
\end{array}
$$

$\hat{w}_{k}(t):=\int_{t-\tau_{k}+\varsigma}^{t} x(\nu) \mathrm{d} \nu+\int_{t-\varsigma}^{t} w(\nu) \mathrm{d} \nu$, and $\hat{\bar{w}}_{s}(t)=\int_{t-\bar{\tau}_{s}+\varsigma}^{t} x(\nu) \mathrm{d} \nu+\int_{t-\varsigma}^{t} w(\nu) \mathrm{d} \nu$, is such that $x(t) \rightarrow x^{*}$ as $t \rightarrow \infty$.

Corollary 2. Consider (3) satisfying Assumption 1, and a given $x^{*} \in \mathbb{R}^{n}$. If there exist $\gamma \in \Gamma$ and symmetric matrices $P_{1}, S_{k}, \bar{S}_{s} \in \mathbb{R}^{n \times n}, k=1, \ldots, N_{1}, s=1, \ldots, N_{2}$, such that (5) holds,

$$
P_{1}, S_{k}, \bar{S}_{s}>0, \quad \text { and } \bar{M}(\gamma):=\left[\begin{array}{cc}
\Phi_{1} & \Phi_{2} \\
* & \Phi_{3}
\end{array}\right]<0,
$$

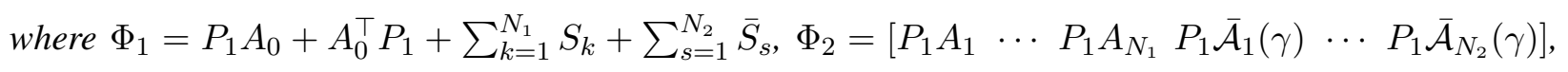
and $\Phi_{3}=-\operatorname{diag}\left(Q_{1}, \ldots, Q_{N_{1}}, \bar{Q}_{1}, \ldots, \bar{Q}_{N_{2}}\right)$. Then, (3) in closed-loop with the switching controller

$$
\begin{gathered}
\sigma(t) \in \underset{j \in\{1, \ldots, N\}}{\arg \min }\left(w(t)-x^{*}\right) P_{1}\left[\sum_{s=1}^{N_{2}} \mathcal{A}_{s, j} x\left(t-\bar{\tau}_{s}+\varsigma\right)+B_{j}\right], \\
w(t)=\mathrm{e}^{A_{0} \varsigma} x(t)+\int_{-\varsigma}^{0} \mathrm{e}^{-A_{0} \eta}\left[\sum_{k=1}^{N_{1}} A_{k} x\left(t-\tau_{k}+\varsigma+\eta\right)+\right. \\
\left.\sum_{s=1}^{N_{2}} \mathcal{A}_{s, \sigma(t+\eta)} x\left(t-\bar{\tau}_{s}+\varsigma+\eta\right)+B_{\sigma(t+\eta)}\right] \mathrm{d} \eta,
\end{gathered}
$$

is such that $x(t) \rightarrow x^{*}$ as $t \rightarrow \infty$. 
The proof of Theorem 2 is analogous to the proof of Theorem 1, but considering the LyapunovKrasovskii functional [32]

$$
\begin{array}{r}
V\left(z_{t}\right)=\hat{\zeta}^{\top} P \hat{\zeta}+\sum_{k=1}^{N_{1}} \int_{t-\tau_{k}}^{t}\left(\nu-t+\tau_{k}\right) z^{\top}(\nu) R_{k} z(\nu) \mathrm{d} \nu+ \\
\sum_{s=1}^{N_{2}} \int_{t-\bar{\tau}_{s}}^{t}\left(\nu-t+\bar{\tau}_{s}\right) z^{\top}(\nu) \bar{R}_{s} z(\nu) \mathrm{d} \nu+ \\
\sum_{s=1}^{N_{2}} \int_{t-\bar{\tau}_{s}}^{t} z^{\top}(\nu) \bar{S}_{s} z(\nu) \mathrm{d} \nu+\sum_{k=1}^{N_{1}} \int_{t-\tau_{k}}^{t} z^{\top}(\nu) S_{k} z(\nu) \mathrm{d} \nu,
\end{array}
$$

where $\hat{\zeta}(t)=\left[z^{\top}(t), \hat{z}_{1}^{\top}(t), \ldots, \hat{z}_{N_{1}}^{\top}(t), \hat{\bar{z}}_{1}^{\top}(t), \ldots, \hat{\bar{z}}_{N_{2}}^{\top}(t)\right]^{\top}, \hat{z}_{k}(t)=\int_{t-\tau_{k}}^{t} z(\nu) \mathrm{d} \nu, \hat{\bar{z}}_{s}(t)=\int_{t-\bar{\tau}_{s}}^{t} z(\nu) \mathrm{d} \nu$,

$$
P=\left[\begin{array}{cc}
P_{1} & \Pi_{11} \\
* & \bar{\Pi}_{22}
\end{array}\right]
$$

and $\bar{\Pi}_{22}=\Pi_{22}-\operatorname{diag}\left(\tau_{1}^{-1} S_{1}, \ldots, \tau_{N_{1}}^{-1} S_{N_{1}}, \bar{\tau}_{1}^{-1} \bar{S}_{1}, \ldots, \bar{\tau}_{N_{2}}^{-1} \bar{S}_{N_{2}}\right)$. For the proof of Corollary 2, set in (16) $P_{2, k}=\bar{P}_{2, s}=P_{3, k}=\bar{P}_{3, s}=R_{k}=\bar{R}_{s}=0$.

\section{EXAMPLES}

In the examples of this section the LMIs were solved in MATLAB by using the solver SeDuMi [34] version 1.3, and the toolbox YALMIP [35]. For the simulations, we used Simulink with the fixed-step Euler integration method.

\section{A. Delay-independent controller}

Here we consider the example given in [26] where the controller is designed by considering delayindependent conditions. Thus, we change the delay in the state (twice the original) to show that the same controller works. Consider (1) with $n=2, m=2, N_{1}=0, N_{2}=1, \tau=2, \varsigma=1 / 2$, and

$$
\begin{gathered}
A_{0}=\left[\begin{array}{cc}
-3 & 1 \\
2 & -5
\end{array}\right], \quad A_{1,1}=\left[\begin{array}{ll}
4 & 2 \\
1 & 6
\end{array}\right], \\
A_{1,2}=\left[\begin{array}{ll}
1 & 1 \\
2 & 2
\end{array}\right], \quad B=\left[\begin{array}{ll}
3 & 1 \\
1 & 2
\end{array}\right] .
\end{gathered}
$$

The control components are assumed to be on-off, i.e. $u_{i}: \mathbb{R} \rightarrow\{0,1\}, i=1,2$, hence $U=\left\{u^{1}, \ldots, u^{4}\right\}$ where $u^{1}=\left[\begin{array}{ll}0 & 0\end{array}\right]^{\top}, u^{2}=\left[\begin{array}{ll}1 & 0\end{array}\right]^{\top}, u^{3}=\left[\begin{array}{ll}0 & 1\end{array}\right]^{\top}$, and $u^{4}=\left[\begin{array}{ll}1 & 1\end{array}\right]^{\top}$. It is important to mention that the trajectories of this model diverge by maintaining any of the inputs on. If we choose $x^{*}=[22]^{\top}$, then for $\gamma=\frac{1}{30}\left[\begin{array}{llll}14 & 4 & 12 & 0\end{array}\right]^{\top}$ we have that the LMIs in (9) hold with

$$
P_{1}=\frac{1}{10}\left[\begin{array}{ll}
4.8581 & 1.1108 \\
1.1108 & 2.6861
\end{array}\right], S=\left[\begin{array}{cc}
1.1122 & -0.0054 \\
-0.0054 & 1.1990
\end{array}\right] \text {. }
$$



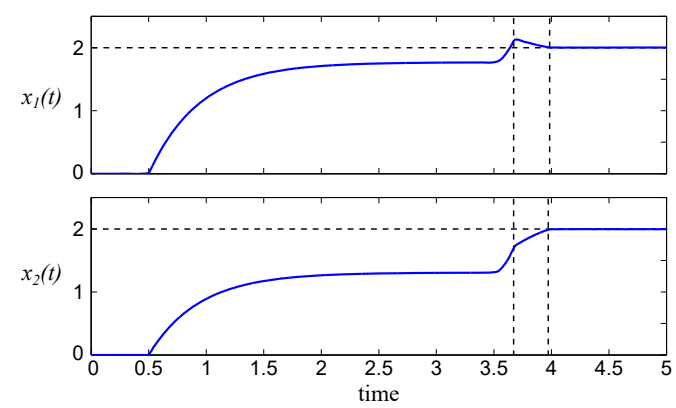

Fig. 1. States of the system.

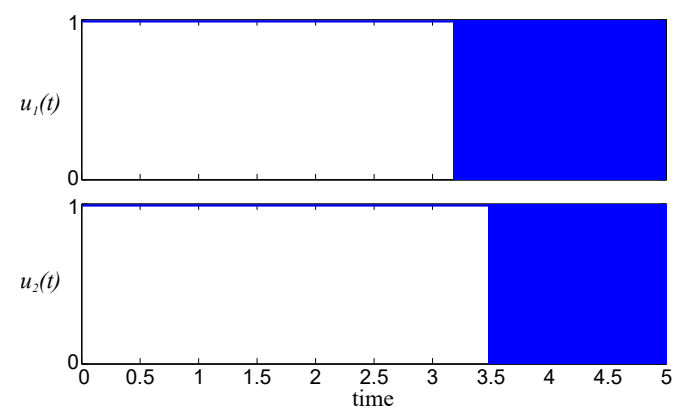

Fig. 2. Control signals.

The initial conditions were chosen as $x(t)=0, u(t)=0$ for all $t \leq 0$, and the integration step was equal to $0.1 \mathrm{~ms}$. Fig. 1 shows the system's states converging to the reference point. The vertical doted lines indicate the time when the delayed inputs start to switch in a high-frequency regime. Such a switching can be appreciated in the nondelayed control signals shown in Fig. 2.

It is important to mention that for this example it can also be used the controller with delay-dependent conditions given in Theorem 1. However, note that (10) is simpler than (8).

\section{B. Delay-dependent controller}

Consider again (1) with $n=2, m=2, N_{1}=0, N_{2}=1, \tau=1 / 3, \varsigma=1 / 6$, and

$$
\begin{gathered}
A_{0}=\frac{1}{20}\left[\begin{array}{cc}
1 / 3 & 1 / 4 \\
1 / 5 & 1 / 2
\end{array}\right], \quad A_{1,1}=\left[\begin{array}{cc}
-5 & 2 \\
1 & 1
\end{array}\right], \\
A_{1,2}=\left[\begin{array}{cc}
1 & 1 \\
2 & -4
\end{array}\right], \quad B=\left[\begin{array}{cc}
1 & 1 \\
1 / 2 & 3 / 2
\end{array}\right] .
\end{gathered}
$$

Note that in this case $A_{0}$ is not a Hurwitz matrix, therefore, the controller given in Corollary 1 cannot be used. 

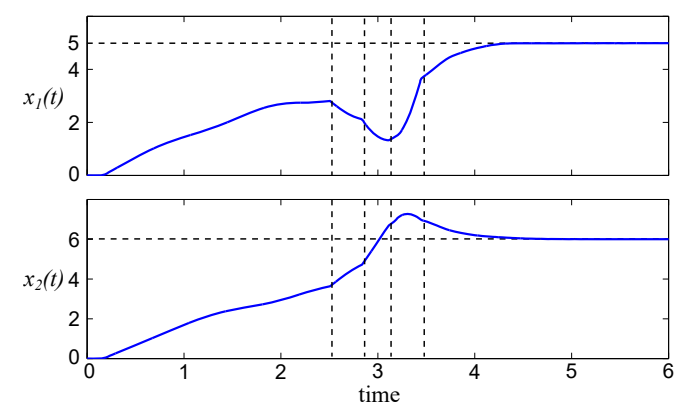

Fig. 3. States of the system.

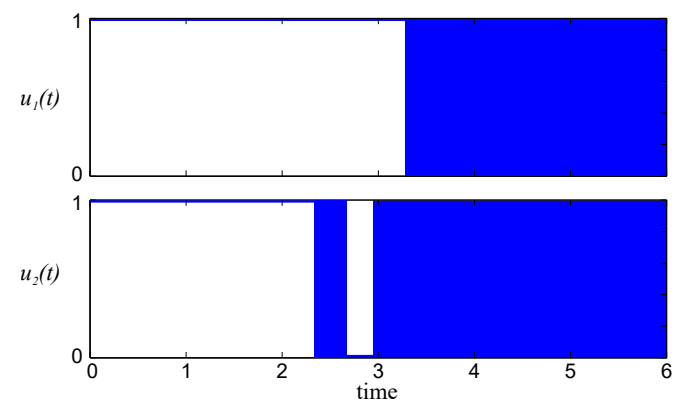

Fig. 4. Control signals.

The control components are assumed to be as in the previous example. If we choose $x^{*}=[56]^{\top}$, then for $\gamma=\frac{1}{30}\left[\begin{array}{llll}8.500 & 10.9479 & 10.5521 & 0\end{array}\right]^{\top}$ we have that the LMIs in (7) hold with

$$
\begin{gathered}
P_{1}=\left[\begin{array}{ll}
1.0731 & 0.6971 \\
0.6971 & 1.3689
\end{array}\right], \quad S=\left[\begin{array}{cc}
0.4933 & -0.0723 \\
-0.0723 & 0.4668
\end{array}\right], \\
R=\left[\begin{array}{cc}
1.2366 & -0.3719 \\
-0.3719 & 1.0906
\end{array}\right], P_{2}=\left[\begin{array}{cc}
-0.7578 & 0.1262 \\
0.1287 & -0.7159
\end{array}\right], \\
P_{3}=\left[\begin{array}{cc}
0.6463 & -0.4995 \\
-0.4995 & 0.4505
\end{array}\right],
\end{gathered}
$$

The initial conditions were chosen as $x(t)=0, u(t)=0$ for all $t \leq 0$, and the integration step was equal to $0.1 \mathrm{~ms}$. Fig. 3 shows the system's states converging to the reference point. The vertical doted lines indicate the times when the delayed inputs start or stop switching in a high-frequency regime. Such a switching can be appreciated in the nondelayed control signals shown in Fig. 4. 


\section{CONCLUSION}

The controller developed in this paper can be used in a wide set of bilinear systems where the delay cannot be neglected. The conceptual simplicity of the controller let it to be modifiable by changing the Lyapunov-Krasovskii functional. An important potential application of this controller is in the field of turbulent flow control systems, since it is suitable for the kind of models and on-off actuators used in some flow control applications, see e.g. [24].

Future work: There are several aspects that can be considered to improve or extend the control approach used in this paper. We can mention, e.g. robustness analysis, consideration of time varying delays, or the presence of several delays in the input.

\section{REFERENCES}

[1] R. R. Mohler, Bilinear Control Processes: With Applications to Engineering, Ecology, and Medicine. New York: Academic Press, 1973.

[2] P. M. Pardalos and V. A. Yatsenko, Optimization and Control of Bilinear Systems. New York: Springer, 2008.

[3] A. Krener, "Bilinear and Nonlinear Realizations of Input-Output Maps," SIAM Journal on Control, vol. 13, no. 4, pp. 827-834, 1975.

[4] D. Elliott, Bilinear Control Systems. Dordrecht, Netherlands: Springer, 2009.

[5] N. N. Krasovskii, Stability of Motion. Stanford, CA: Stanford Univ. Press, 1963.

[6] J.-P. Richard, "Time-delay systems: an overview of some recent advances and open problems," Automatica, vol. 39, no. 10, pp. 1667-1694, 2003.

[7] E. Fridman, Introduction to Time-Delay Systems. Birkhäuser Basel, 2014.

[8] H. Sira-Ramirez and R. Silva-Ortigoza, Control Design Techniques in Power Electronics Devices. London: Springer-Verlag, 2006.

[9] P. Beater, Pneumatic Drives. Berlin: Springer-Verlag, 2007.

[10] P. Bolzern and W. Spinelli, "Quadratic stabilization of a switched affine system about a nonequilibrium point," in Proceedings of the 2004 American Control Conference, June 2004, pp. 3890-3895 vol. 5.

[11] G. S. Deaecto, J. C. Geromel, F. S. Garcia, and J. A. Pomilio, "Switched affine systems control design with application to DC-DC converters," IET Control Theory Applications, vol. 4, no. 7, pp. 1201-1210, July 2010.

[12] L. Hetel and E. Fridman, "Robust Sampled - Data Control of Switched Affine Systems," IEEE Transactions on Automatic Control, vol. 58, no. 11, pp. 2922-2928, Nov 2013.

[13] L. Hetel, M. Defoort, and M. Djemaï, "Binary Control Design for a Class of Bilinear Systems: Application to a Multilevel Power Converter," IEEE Transactions on Control Systems Technology, vol. 24, no. 2, pp. 719-726, March 2016.

[14] Z. Kader, C. Fiter, L. Hetel, and L. Belkoura, "Stabilization of switched affine systems with disturbed state-dependent switching laws," International Journal of Robust and Nonlinear Control, vol. 28, no. 2, pp. 582-595, 2018.

[15] W. Jiang, E. Fridman, A. Kruszewski, and J. Richard, "Switching controller for stabilization of linear systems with switched time-varying delays," in Proceedings of the 48h IEEE Conference on Decision and Control (CDC) held jointly with 2009 28th Chinese Control Conference, Dec 2009, pp. 7923-7928.

[16] L. Vu and K. A. Morgansen, "Stability of time-delay feedback switched linear systems," IEEE Transactions on Automatic Control, vol. 55, no. 10, pp. 2385-2390, Oct 2010. 
[17] F. Mazenc, S. Ahmed, and H. Özbay, "State feedback stabilization of switched systems with delay: Trajectory based approach,” in 2017 American Control Conference (ACC), May 2017, pp. 4540-4543.

[18] Y. Zhang, X. Liu, and X. Shen, "Stability of switched systems with time delay," Nonlinear Analysis: Hybrid Systems, vol. 1, no. 1, pp. $44-58,2007$.

[19] K. Moezzi, L. Rodrigues, and A. G. Aghdam, "Stability of uncertain piecewise affine systems with time delay: delaydependent Lyapunov approach,” International Journal of Control, vol. 82, no. 8, pp. 1423-1434, 2009.

[20] M. S. Mahmoud, Switched Time-Delay Systems. New York: Springer US, 2010.

[21] C. Fiter and E. Fridman, "Stability of Piecewise Affine Systems with State-Dependent Delay, and Application to Congestion Control," in 52nd IEEE Conference on Decision and Control, Dec 2013, pp. 1572-1577.

[22] M. Feingesicht, C. Raibaudo, A. Polyakov, F. Kerhervé, and J.-P. Richard, "A bilinear input-output model with statedependent delay for separated flow control," in 2016 European Control Conference (ECC), June 2016, pp. 1679-1684.

[23] M. Feingesicht, "Nonlinear active control of turbulent separated flows: Theory and experiments," Ph.D. dissertation, Centrale Lille, France, 2017.

[24] M. Feingesicht, A. Polyakov, F. Kerhervé, and J.-P. Richard, "SISO model-based control of separated flows: Sliding mode and optimal control approaches," International Journal of Robust and Nonlinear Control, vol. 27, no. 18, pp. 5008-5027, 2017.

[25] T. Sanchez, A. Polyakov, J.-P. Richard, and D. Efimov, "A robust Sliding Mode Controller for a class of SISO bilinear delayed systems," in 15th International Workshop on Variable Structure Systems (VSS), July 2018, pp. $126-131$.

[26] T. Sanchez, A. Polyakov, L. Hetel, and E. Fridman, "A switching controller for a class of MIMO bilinear time-delay systems," in 2018 IEEE International Conference on the Science of Electrical Engineering (ICSEE), Dec 2018.

[27] J. K. Hale and S. M. Verduyn Lunel, Introduction to Functional Differential Equations. New York: Springer-Verlag, 1993.

[28] V. Kolmanovskii and A. Myshkis, Introduction to the Theory and Applications of Functional Differential Equations. Dordrecht, Netherlands: Springer, 1999.

[29] R. P. Agarwal, L. Berezansky, E. Braverman, and A. Domoshnitsky, Nonoscillation Theory of Functional Differential Equations with Applications. New York: Springer-Verlag, 2012.

[30] Q.-C. Zhong, “On Distributed Delay in Linear Control Laws-Part I: Discrete-Delay Implementations,” IEEE Transactions on Automatic Control, vol. 49, no. 11, pp. 2074-2080, Nov 2004.

[31] W. Michiels and H. U. Ünal, "Evaluating and Approximating FIR Filters: An Approach Based on Functions of Matrices," IEEE Transactions on Automatic Control, vol. 60, no. 2, pp. 463-468, Feb 2015.

[32] E. Fridman and L. Shaikhet, "Simple LMIs for stability of stochastic systems with delay term given by Stieltjes integral or with stabilizing delay," Systems \& Control Letters, vol. 124, pp. 83 - 91, 2019.

[33] P. Pepe, "On Liapunov-Krasovskii functionals under Carathéodory conditions," Automatica, vol. 43, no. 4, pp. 701-706, 2007.

[34] J. F. Sturm, "Using SeDuMi 1.02, a MATLAB toolbox for optimization over symmetric cones," Optimization Methods and Software, vol. 11-12, pp. 625-653, 1999, version 1.05 available from http: / / fewcal.kub.nl/sturm.

[35] J. Löfberg, "YALMIP : A Toolbox for Modeling and Optimization in MATLAB," in 2004 IEEE International Conference on Robotics and Automation (IEEE Cat. No.04CH37508), Sept 2004, pp. 284-289. 